\begin{tabular}{|c|c|}
27 février 1897 - Naissance à Paris (France) \\
1930 & $\begin{array}{l}\text { Nommé astronome à l'Observatoire de Meudon } \\
\text { Invention du Coronographe } \\
\text { Invention du filtre de Lyot }\end{array}$ \\
1933 & $\begin{array}{l}\text { Membre de l'Académie des Sciences } \\
\text { Gold Medal de la Royal Astronomical Society } \\
1939\end{array}$ \\
1947 & $\begin{array}{l}\text { Bruce Medal } \\
\text { Draper Medal }\end{array}$ \\
2 avril 1951 & - Mort au Caire (Egypte) \\
\hline
\end{tabular}

\title{
Bernard Ferdinand Lyot
}

\section{Riad Haidar}

haidar@onera.fr

\begin{abstract}
Astronome français, ingénieur de talent dové d'un sens physique remarquable, Bernard Lyot est surtout connu pour avoir révolutionné l'instrumentation solaire avec l'invention du coronographe, ainsi que pour ses photographies spectroscopiques de la couronne solaire.
\end{abstract}

B ernard Ferdinand Lyot naît le 27 février 1897 à Paris, dans une famille aisée. Son père, chirurgien des hôpitaux, décède quelques années plus tard. Le jeune Bernard montre très vite de grandes qualités manuelles et un goût prononcé pour les sciences. À seize ans, il se passionne pour l'astronomie et installe un petit observatoire, avec un télescope de 100 mm, au-dessus de la maison familiale.

Élève brillant et très actif, il fait ses classes au lycée Janson de Sailly à Paris et intègre en 1914 l'École Supérieure d'Électricité. Il y fait la connaissance d'Alfred Pérot [1863-1925], alors professeur à l'École polytechnique et physicien attaché à l'Observatoire de Meudon. Pérot, séduit par la vivacité d'esprit de l'étudiant, lui propose de devenir préparateur d'expériences pour ses cours de physique; Lyot s'en acquitte avec talent, et secondera ainsi Pérot pendant plusieurs années.

Dans une France qui s'embourbe dans la Grande Guerre, Bernard Lyot, fraichement diplômé de Supélec dont il est le major de promotion, rejoint en 1917 le laboratoire de télégraphie militaire dirigé par le Général Ferrié. Là, il confirme et développe un talent rare d'inventeur, capable d'innovation en s'écartant des méthodologies et des paradigmes habituels. On lui doit notamment les procédés originaux de radionavigation pour la marine, qui seront recyclés plus tard pour l'aviation.

Intronisé par Pérot, il fait la connaissance de Charles Fabry [1867-1945], et surtout d'Henri Deslandres [1853-1948] qui lui ouvre l'accès aux laboratoires de l'Observatoire de Meudon. C'est la réalisation d'un rêve d'enfant. En 1920, il obtient un poste d'assistant qui lui permet de poursuivre ses travaux expérimentaux et de compléter sereinement sa formation universitaire.

\section{Lyot, I'astronome}

Bernard Lyot s'intéresse à la signature polarimétrique de la lumière réfléchie par la surface des planètes. C'est un sujet plein de promesses car cette propriété permet d'identifier à distance la composition des sols. Mais les mesures restent extrêmement difficiles, en raison surtout de la précision insuffisante des instruments disponibles... Voici un défi à la hauteur de l'inventivité de Lyot : en 1922, s'inspirant du polariscope de Savart, il conçoit et met au point un polarimètre visuel à franges, un outil dix fois plus sensible que les meilleurs instruments de l'époque. C'est un véritable tour de force, qu'il réussit avec ce que certains qualifient d'une économie artistique de moyens; cela lui confère surtout un avantage expérimental qui lui permet de détecter et mesurer, pour la première fois, le taux de polarisation très faible des planètes. Il en étudie la variation avec l'angle de phase, et démontre qu'il existe une grande similitude entre les courbes de polarisation de Mercure, de la Lune et de Mars, et celle des poussières volcaniques; ou entre celle de Vénus et celle des nuages. II en fera le sujet de sa thèse, soutenue le 18 juin 1929 sous le titre de Recherche sur la polarisation de la lumière des planètes et de quelques substances terrestres. Son doctorat en poche, il décroche un poste d'astronome en 1930. 


\section{OPTICIENS CÉLÈBRES}

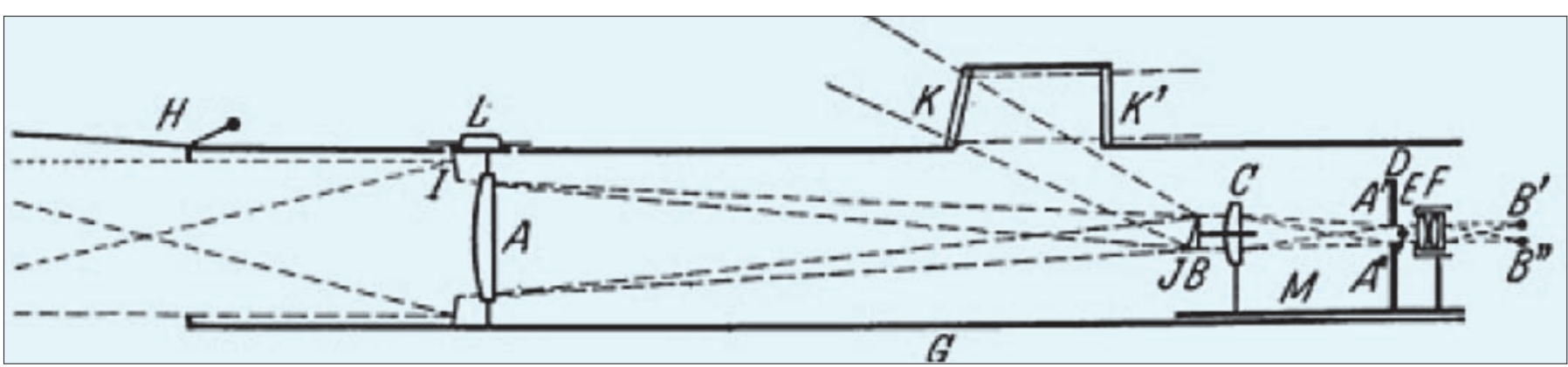

Schéma du coronographe (in B. Lyot, Zeitschrift für Astrophysik, Bd. 5, p. 73-95, 1932).

Sur une suggestion de Fabry, Lyot décide d'utiliser son polarimètre pour étudier la couronne solaire en dehors d'une éclipse. En effet la lumière de la couronne est polarisée par diffusion, une propriété remarquable, connue depuis le $19^{e}$ siècle et qui pourrait servir de signature distinctive pour isoler le flux coronal - mais que nul encore n'est parvenu à exploiter efficacement. Pourtant les tentatives furent nombreuses: Huggins [1824-1910], Deslandres ou encore Hale [1868-1938]... Lyot comprend que la limite principale vient de la lumière instrumentale parasite, qui noie littéralement le flux utile dans l'énorme flux photosphérique diffusé. II parvient à la réduire grâce à une série d'astuces instrumentales et au choix soigné d'optiques taillées et polies dans des matériaux d'une pureté irréprochable.

\section{Le coronographe}

En 1930, son coronographe au point, Bernard Lyot se rend au Pic du Midi. Son instrument, d'une précision alors inégalée, tient tout entier dans un sac à dos et peut être transporté à dos d'homme. Eł cela, précisément, est spectaculaire : sans avoir inventé ni découvert de nouveau principe physique, disposant d'éléments largement connus et de concepts maîtrisés par tous, sans recourir aux télescopes monumentaux qui passent pour être I'arme absolve universelle, il parvient à repousser les limites de sensibilité en bricolant lui-même, avec des moyens modestes, un objet qui révolutionne l'instrumentation solaire. Et il réussit là où d'autres, brillants et également obstinés, ont pourtant échové. Car l'expérience du Pic du Midi est un plein succès : pour la première fois la couronne solaire, si riche d'enseignements, peut être observée et photographiée en absence d'éclipse. Ses résultats suscitent l'intérêt immédiat de la communauté scientifique, et lui valent le Prix Louis Ancel de la Société Française de Physique (1931) et le Prix Janssen de la Société Astronomique de France (1932).

Lyot ne cesse plus, dès lors, de perfectionner son instrument. À l'aide d'un spectroscope placé au foyer du coronographe, il met en évidence certaines signatures spectrales particulières. Pour réduire les temps de pose, il conçoit un filtre monochro matique d'un type nouveau, basé sur l'interférence de l'onde ordinaire et de l'onde extraordinaire à la sortie d'un cristal biréfringent uniaxe - désormais connu comme le filtre de Lyot.
Il peut ainsi étudier la raie verte, et montre que la couronne intérieure tourne avec la photosphère. II invente ensuite le coronomètre photoélectrique qui permet une observation sur plaine, sous des cieux ordinaires et moins purs qu'en haute altitude. En 1948, cet expérimentateur infatigable travaille sur le contraste de phase avec Maurice Françon [1913-1996].

\section{La gloire}

Universellement connu pour avoir révolutionné l'instrumentation solaire, Bernard Lyot reçoit pratiquement tous les honneurs. Il est élu membre de l'Académie des Sciences en 1939, et décroche la même année la Gold Medal de la Royal Astronomical Society de Londres. En 1941, le Franklin Institute lui décerne la Haward N. Potts Medal. II est nommé astronome en chef de l'Observatoire de Meudon en 1943, et obtient la Légion d'Honneur en 1946. En 1947, I'Astronomical Society of the Pacific lui remet la Catherine Wolf Bruce Medal pour couronner I'ensemble de ses travaux. En 1951, il reçoit la prestigieuse Draper Medal de la National Academy of Sciences des ÉtatsUnis.

En 1951, en prévision de l'éclipse du 25 février 1952, il se rend au Caire, avec un spectrographe à fente circulaire de son invention, pour étudier le spectre d'émission de la basse couronne. Cette campagne de mesures se révèle effroyablement pénible. Lyot a du mal à mobiliser ses troupes : il épuise ses forces à mettre au point, seul ou pratiquement, toutl'échafaudage expérimental. Le 2 avril, sur la route qui mène à l'Observatoire de Hélouan, il succombe à une crise cardiaque.

Lyot a alors 55 ans. Il laisse le souvenir d'un homme modeste et simple, bricoleur élégant et habile expérimentateur, irrésistiblement attiré par l'étude des phénomènes réputés impossibles à détecter. En astronomie solaire, ce furent autant de défis que son ingéniosité, à la fois conceptuelle et manuelle, parvint à relever.

\section{Références}

[1] A. Danjon, Bernard Lyot, Annales d'Astrophysique, n²2 (1952).

[2] L. d'Azambuj, L'œuvre de Bernard Lyot, L'Astronomie, 66, 267-277 (1952). 